\title{
Studying equilibria of polymers in solution by direct molecular dynamics simulations: poly(n-isopropylacrylamide) in water as a test case
}

Edder J. García and Hans Hasse ${ }^{\mathrm{a}}$

Laboratory of Engineering Thermodynamics (LTD), University of Kaiserslautern, ErwinSchrödinger-Str. 44, 67663 Kaiserslautern, Germany

\begin{abstract}
It is well known that studying equilibria of polymers in solution by atomistic simulations is computationally demanding as a large phase space has to be adequately sampled. Nevertheless, direct molecular dynamics (MD) simulations are often used for this purpose in the literature. To assess whether such approach is adequate, we have conducted a case study for a polymer + solvent system that has been commonly studied with direct MD simulations by many authors: poly(nisopropylacrylamide) (PNIPAM) in water. The total simulation time of the present study is much longer than that typically used in MD simulations of that system. A NIPAM chain of 30 monomers was studied in explicit water at $295 \mathrm{~K}$. Three initial configurations were used. For each configuration, five replicas were run for 1000 ns. The statistical analysis of our data shows that the equilibration time is of the order of 600 - $700 \mathrm{~ns}$ and that the remaining time for the production run is not sufficient to sample the equilibrium state adequately. These results underpin the well-known difficulty of sampling equilibrium states of polymers in solution with direct MD simulations and the need for a careful interpretation of results of such studies. The problem with the unsatisfactory sampling persists despite the increasing available computing power. Therefore, enhanced sampling techniques and workarounds, such as simplified scenarios or coarse-graining, remain important.
\end{abstract}

\section{Introduction}

The present study deals with molecular dynamics (MD) simulation studies of equilibrium properties of polymers in solutions. Such simulations are computationally expensive as the configurational space that has to be sampled is large. To save computing time, many authors conduct MD simulations using a single polymer chain, i.e., an infinitely diluted polymer. The present discussion is limited to this case.

Depending on the solvent and the chosen conditions, such as the temperature and $\mathrm{pH}$ value, the equilibrium conformations of a polymer chain in a solvent can

\footnotetext{
${ }^{a}$ e-mail: hans.hasse@mv.uni-kl.de
} 
differ strongly. Usually, two states are distinguished: the coil state (loose, elongated configuration), which is characteristic of a polymer in a good solvent, and the globule state (compact configuration), obtained in a poor solvent. Upon varying the nature of the solvent, temperature or $\mathrm{pH}$ value, a transition between these equilibrium states may occur, which is called coil-globule transition. The coil-globule transition is closely related to phenomenological properties of the polymer solution, namely the polymer solubility and the polymer's osmotic virial coefficients [1-3].

Predicting the coil-globule transition based on atomistic force fields by MD simulations is highly attractive, and there are many reports on this in the literature, e.g. [4-15]. The present work focusses on solutions of poly(n-isopropylacrylamide) (PNIPAM) in water, in which the coil-globule transition occurs at about $305 \mathrm{~K}$ [16-18]. This transition is particularly important as it drives the swelling transition of PNIPAM hydrogels. It has therefore been studied by many authors using MD simulations based on atomistic force fields, see Table 1. Usually, a NIPAM chain of 30 monomers is studied $[4-7,19]$, which is computationally acceptable and does not lead to dominating effects of the end-groups. Only simulations with explicit water are considered here. Many force fields are available for modeling the polymer and water molecules (see Table 1). As the present study is concerned with simulation issues and not with physical modeling, the choice of the force field is not discussed further here. The force field that was chosen for the present study is specified below. It can be assumed that the basic findings of the present work are independent of the choice of the force field for PNIPAM or water.

For determining the coil-globule transition in molecular simulations of single polymer chains, usually the radius of gyration $R_{\mathrm{g}}$ of the polymer is monitored, and threshold values for $R_{\mathrm{g}}$ are chosen to distinguish the coil and the globule state. The definition of the thresholds is somehow arbitrary, and some 'intermediate' state between the coil and globule state can be introduced [10]. At equilibrium, $R_{\mathrm{g}}$ has a certain probability distribution $P\left(R_{\mathrm{g}}\right)$. The macroscopic value $\left\langle R_{\mathrm{g}}\right\rangle$ is the probability-weighted average. In MD simulations, the macroscopic $R_{\mathrm{g}}$ value is approximated by averaging the results for each time step of the production run, discarding the results from the equilibration at the beginning of the simulation.

For obtaining a reasonable estimate of the equilibrium value of $R_{\mathrm{g}}$, both the equilibration and the sampling time have to be long enough. It is well known that the equilibration of systems with polymers requires long simulation time [20-22]. For instance in MD simulations of small proteins containing from 10 to 80 amino acids, the time for obtaining the native structure (i.e., the equilibrium conformation) starting from a random coil conformation has been found to vary between $2 \mu$ s and 643 $\mu \mathrm{s}[23,24]$. Comparing these numbers to the simulation times of the previous MD studies of PNIPAM chains as reported in Table 1 casts doubts on whether information on the equilibrium can be obtained from these studies in which the simulation time never exceeded $1.0 \mu \mathrm{s}$. These doubts are strengthened by the fact that in most of these simulations only very few transitions between the globule and coil states were observed, if any were observed at all. Even the results of the longest available simulations $[4,7,13,25]$ are not entirely conclusive regarding the equilibrium conformation of PNIPAM in water. The authors of [7] and [13] have explicitly mentioned the problems of sampling the equilibrium state in their studies. In a study of cononsolvency of PNIPAM in mixtures of water and methanol, Dalgicdir et al. [26] conducted 200 independent MD simulations for a 40mer-NIPAM in pure water (as well as for several mixtures of water and methanol). The initial configurations of the 200 simulations were different, and the simulation time was $100 \mathrm{~ns}$ in each run. No statistical analysis of the results of the 200 runs was presented to check the equilibration. The reason for the long time to reach equilibrium is that the polymer can get stuck in a longlived metastable configuration. It is well known that MD is an inefficient method for 
exploring the conformation space of flexible molecules with important energy barriers associated with internal degrees of freedom [27]. It has been shown that even for simple molecules, such as cyclohexane or cycloheptadecane, direct analysis of MD data for obtaining information on equilibrium conformations may be difficult $[28,29]$. Studies of the intramolecular transitions by MD typically require high temperatures to explore a large number of conformers $[29,30]$.

A prerequisite for calculating the equilibrium value of $R_{\mathrm{g}}$ is to distinguish between the equilibration and the sampling period. Several methods have been proposed for assessing whether the studied system is sufficiently equilibrated to start sampling [31-35]. At equilibrium, the average $R_{\mathrm{g}}$ must be stationary, i.e., the average $R_{\mathrm{g}}$ does not increase or decrease over time. Therefore, to identify when the equilibrium is reached, it is necessary to detect trends in the data. A convenient method for such analysis is the Mann-Kendall (MK) test $[36,37]$ because it does not require a specific distribution of the data or the residuals. In the present work, the MK test is used to determine the equilibration time by detecting trends in the $R_{\mathrm{g}}$ data obtained from MD simulations [31,38].

The MK test assumes that the data is uncorrelated. Uncorrelated data can be generated using $\mathrm{MD}$ either by dividing a single trajectory into blocks or by running several replicas in parallel. In this work, we run fifteen independent MD simulations in parallel and perform the MK test over the average $R_{\mathrm{g}}$ over all runs. Parallel replicas allow (1) to improve the configurational sampling [39] and (2) to apply the GelmanRubin statistical test [32] for checking whether single runs are representative of the pooled distribution. The MK test shows that the combined fifteen replicas reach equilibrium at around $670 \mathrm{~ns}$ per replica, i.e., $10.0 \mu \mathrm{s}$ of total simulation time. The Gelman-Rubin test shows that single runs are not representative of the pooled distribution. The results underpin the difficulty of sampling equilibrium states of polymers in solution with direct MD simulations and highlight the necessity of enhanced sampling techniques and workarounds, such as simplified scenarios or coarse-graining, for such studies.

\section{Simulations}

All simulations in the present work were performed with a single, syndiotactic NIPAM chain of 30 monomers. The end groups were capped using a hydrogen site and a $\mathrm{CH}_{3}$ group. A cubic simulation box with a volume of approximately $9.2 \times 9.2 \times 9.2 \mathrm{~nm}^{3}$ was used. The total number of water molecules was 26,345 .

Short pre-equilibration runs were carried out for $50 \mathrm{ps}$ in the $N V T$ ensemble and $200 \mathrm{ps}$ in the $N p T$ ensemble. The temperature was fixed at $295 \mathrm{~K}$ by the Vscaling thermostat [42]. The reasons for the choice of the temperature are given below. The pressure was set to 1 bar by the Berendsen barostat [43] during the $N p T$ pre-equilibration run and the Parrinello-Rahman barostat [44] during the collection of the data. The equations of motion were integrated using the leapfrog algorithm. A spherical cutoff radius of $1.4 \mathrm{~nm}$ was used to truncate non-bonded interactions. Tail corrections were used for the potential energy and pressure. The electrostatic interactions were calculated by the PME method [45], using a grid spacing of 0.12 $\mathrm{nm}$ and a fourth-order interpolation. Periodic boundary conditions were applied in all directions of space.

The OPLS/AA force field [46] was used for NIPAM and the SPC/E model [47] for water. Geometric mixing rules were applied to calculate the Lennard-Jones parameters for unlike polymer-water and polymer-polymer interaction sites. The same force field was used in our earlier study [10]. The force field parameters are presented in the Supplementary Material. 
Table 1: Overview of all-atom simulations of NIPAM in pure water using a single polymer chain.

\begin{tabular}{|c|c|c|c|c|c|}
\hline Year [Ref.] & Time / ns & $\begin{array}{l}\text { Monomers } \\
\text { per chain }\end{array}$ & $\begin{array}{c}\text { Force field } \\
\text { (Polymer-water) }\end{array}$ & $\begin{array}{c}\mathrm{T} \text { range } \\
/ \mathrm{K}\end{array}$ & $\begin{array}{c}\text { Initial } \\
\text { configuration }\end{array}$ \\
\hline $2004[8]$ & 4.4 & 50 & Amber $^{(a)}$-TIP3P & $300-310$ & intermediate \\
\hline $2008[9]$ & 75 & 26 & Amber ff03 ${ }^{(\mathrm{a})}-\mathrm{TIP} 3 \mathrm{P}$ & $302-315$ & coil \\
\hline $2010[10]$ & 40 & 30 & OPLS-SPC/E & $280-360$ & coil \\
\hline $2010[11]$ & 26 & 50 & Amber-94 (a)-TIP3P & $295-310$ & coil \\
\hline $2010[12]$ & 18 & 28 & Gromos 45A3-SPC & $293-323$ & coil \\
\hline $2011[4]$ & 200 & 30 & OPLS-TIP3P & $280-330$ & coil \\
\hline $2012[5]$ & 20 & 30 & PCFF & $278-310$ & coil \\
\hline $2013[6]$ & 20 & 30 & $\mathrm{PCFF}$ & $278-310$ & coil \\
\hline $2016[7]$ & 300 & 30 & $\mathrm{OPLS}^{(\mathrm{a})}-\mathrm{SPC} / \mathrm{E}$ & $280-320$ & $\begin{array}{c}\text { coil } \\
\text { globule }\end{array}$ \\
\hline 2016 [13] & 1000 & 30 & OPLS-TIP4P/2005 & $270-320$ & $\begin{array}{c}\text { coil } \\
\text { intermediate } \\
\text { globule }\end{array}$ \\
\hline $2016[40]$ & 180 & 30 & OPLS $^{(\mathrm{b})}-\mathrm{TIP} 4 \mathrm{P} / 2005$ & $283-323$ & coil \\
\hline $2017[19]$ & 40 & 30 & CHARMM36-TIP3P & $275-325$ & coil \\
\hline $2017[14]$ & 70 & 32 & $\mathrm{OPLS}^{(\mathrm{a})}-\mathrm{SPC} / \mathrm{E}$ & $280-330$ & coil \\
\hline $2017[26]$ & $100^{(\mathrm{c})}$ & 40 & OPLS-SPC/E & 300 & $\begin{array}{c}\text { coil } \\
\text { intermediate } \\
\text { globule }\end{array}$ \\
\hline
\end{tabular}

${ }^{a}$ Modified charges.

b Modifications from Ref. [41].

${ }^{c} 200$ runs of 100 ns each were conducted in pure water.

To allow a time step of $4 \mathrm{fs}$, the bond lengths were constrained using the LINCS algorithm [48] and the degrees of freedom of hydrogen sites were removed by applying virtual sites [49-51]. Bond-stretching vibrations and degrees of freedom involving hydrogens have the highest frequency in the system. If these degrees of freedom are removed, the equations of motion can be integrated with acceptable accuracy using a time step of $4 \mathrm{fs}$, which guarantees at least five integration steps per oscillation. Details on the construction of the virtual sites are given in the Supplementary Material. The application of virtual sites for locating hydrogens has no significant effect on the accuracy of the integration or dynamics [49-51].

The state of the NIPAM chain was characterized by monitoring the radius of gyration $R_{\mathrm{g}}$ every $20 \mathrm{ps}$. As in previous works $[7,10]$, the following thresholds were used: for $R_{\mathrm{g}}<1.2 \mathrm{~nm}$ the oligomer is in the globule state, and for $R_{\mathrm{g}}>1.5 \mathrm{~nm}$ it is in the coil state. The region $1.5 \mathrm{~nm} \geq R_{\mathrm{g}} \geq 1.2 \mathrm{~nm}$ was taken as an intermediate, undefined state. 
The simulations were started from three different initial configurations, a coiled $\left(R_{\mathrm{g}, \text { ini }}=1.80 \mathrm{~nm}\right)$, an intermediate $\left(R_{\mathrm{g}, \text { ini }}=1.20 \mathrm{~nm}\right)$, and a globule $\left(R_{\mathrm{g}, \text { ini }}=0.94\right.$ $\mathrm{nm})$ configuration. The initial configurations were obtained by conducting a short MD simulation in the $N V T$ ensemble at $360 \mathrm{~K}$ starting from the linear conformation of the oligomer. For each initial configuration, a series of five independent simulations were carried out. So in total, fifteen independent simulations, each of $1000 \mathrm{~ns}$, were conducted. All simulations were carried out with Gromacs 5.0 [52,53]. The MD simulations of this work required approximately $340,000 \mathrm{CPU}$ h in total.

For all simulations, the temperature was set to $295 \mathrm{~K}$. A low temperature was preferred as it enhances the sampling problems that we want to study. Furthermore, for low temperatures, previous simulations of NIPAM chains with the force field that was applied in the present work have yielded mixed results regarding the prevailing configuration $[7,13,25]$. For distinctly higher temperatures, the NIPAM chain collapses quickly independently of the chosen initial configuration and then remains in the globule state. Also, $295 \mathrm{~K}$ is close to the experimental transition temperature of PNIPAM, which is the most important property in many applications.

\section{Results, analysis, and discussion}

\subsection{Radius of gyration for single runs}

Fig. 1 shows the radius of gyration of the NIPAM chain as a function of the simulation time for all runs. In Fig. 1, the time resolution was reduced to 1 ns to diminish the fluctuations of the signals and enable a better distinction between the different curves. Results for the five replicas for each of the three different initial configurations are shown in the three panels. Independently of the initial configuration, the NIPAM chain tends to be in the globule state in all runs. There are important differences between the results for the replicas. There are also some unexpected events. For instance, panel (a) of Fig. 1 shows results for one run (red curve) in which the NIPAM, starting from the globule state fully unfolds up to $R_{\mathrm{g}}=2 \mathrm{~nm}$ and then returns to the globule state, while none of the other replicas shows such behavior. Panel (c) shows results for a run (yellow curve) in which the NIPAM, after remaining in the globule state $\left(R_{\mathrm{g}}<\right.$ $1.2 \mathrm{~nm})$ for a long time, quickly unfolds again and reaches the globule state $\left(R_{\mathrm{g}}>\right.$ $1.5 \mathrm{~nm}$ ) shortly before the simulation was stopped.

It is clear that from results like the ones shown in Fig. 1, it is difficult to obtain reliable information on the equilibrium value of $R_{\mathrm{g}}$. It would be practically impossible if only results from a single simulation were available. These difficulties have been pointed out previously by several authors $[4,7,13,25]$, including our own group.

\subsection{Number of coil-to-globule and globule-to-coil transitions}

Fig. 2 shows the total number of coil-to-globule (C-to-G) and globule-to-coil transitions (C-to-G) for the NIPAM chain. The number of C-to-G transitions was determined by counting the shifts of $R_{\mathrm{g}}$ from $R_{\mathrm{g}}>1.5 \mathrm{~nm}$ (coil state) to $R_{\mathrm{g}}<1.2 \mathrm{~nm}$ (globule state). The G-to-C transition was defined analogously. Fig. 2 shows the cumulative number of transitions for the three different initial states. When comparing Figures 1 and 2, it has to be considered that the time resolution of the plots in Fig. 1 is low and not all transitions are easy to discern. Fluctuations between the coil and the globule stop after approximately $570 \mathrm{~ns}$, and the preferred state is clearly the globule. Therefore, after equilibration, only the globule state is sampled. As a consequence, any calculation of the free energy of the coil-globule transition based on such data is practically impossible. 


\subsection{Equilibration time}

Fig. 3 shows the average $R_{\mathrm{g}}$ for the combined 15 runs as a function of the simulation time. The reported numbers are the arithmetic average of all runs for each time $t$.

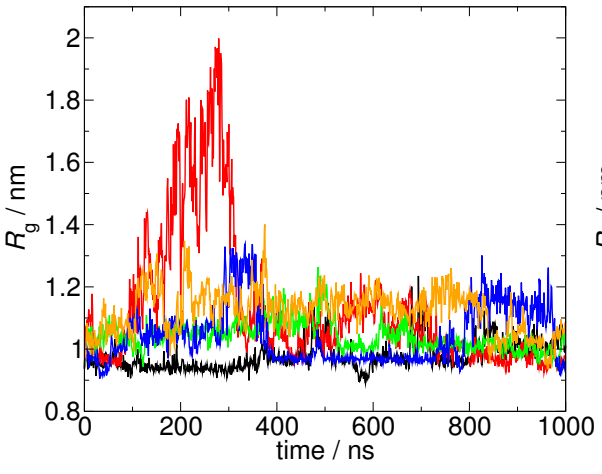

(a)

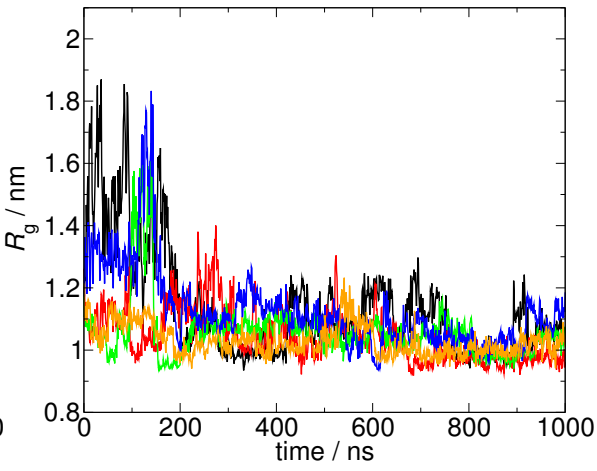

(b)

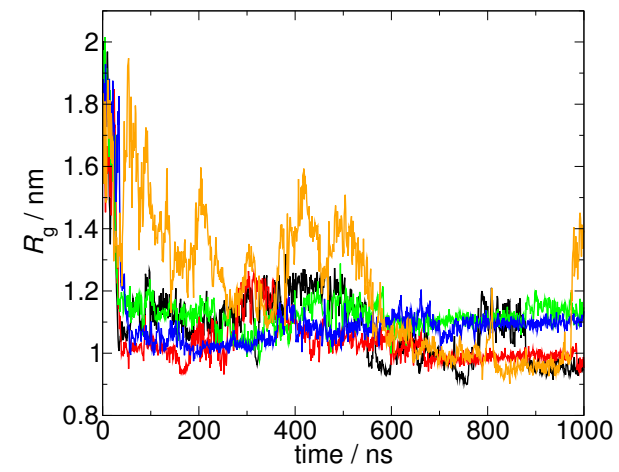

(c)

Fig. 1: Radius of gyration as a function of the simulation time. The simulations were started at (a) $R_{\mathrm{g}, \text { ini }}=0.94 \mathrm{~nm}$, (b) $R_{\mathrm{g}, \text { ini }}=1.20 \mathrm{~nm}$, and (c) $R_{\mathrm{g}, \text { ini }}=1.80 \mathrm{~nm}$.

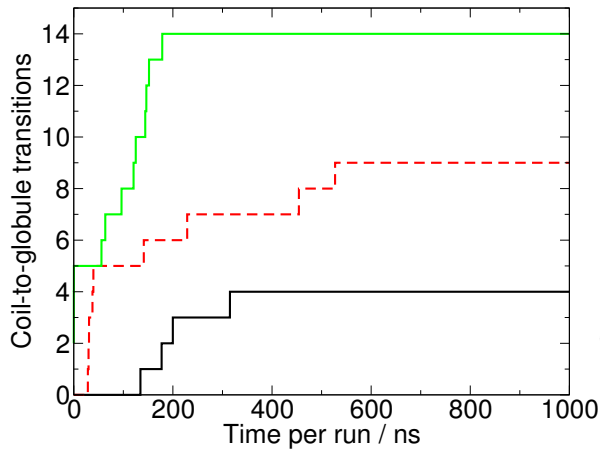

(a)

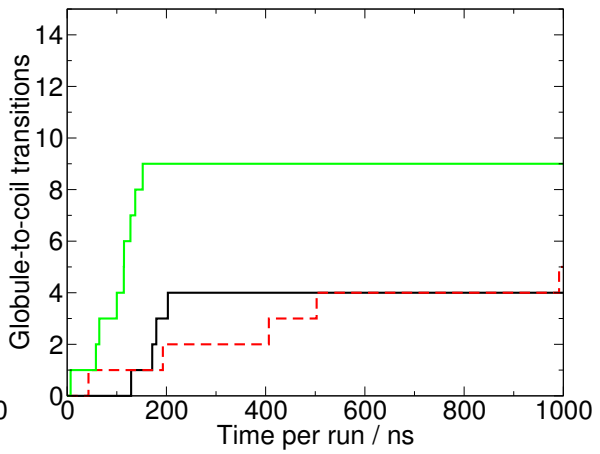

(b)

Fig. 2: Number of (a) coil-to-globule and (b) globule-to-coil transitions as a function of the simulation time per run. Simulations started at $R_{\mathrm{g}, \text { ini }}=0.94 \mathrm{~nm}$ (black line), $R_{\mathrm{g}, \text { ini }}=1.20 \mathrm{~nm}$ (red dashed line), and $R_{\mathrm{g}, \text { ini }}=1.80 \mathrm{~nm}$ (green line). 
Also, results from block averaging with a block size of 5 ns are shown. The signals show asymptotic behavior, i.e., a quasi-stationary state in which the signals only fluctuate is reached after about $650 \mathrm{~ns}$, in good agreement with the analysis of the transitions presented above.

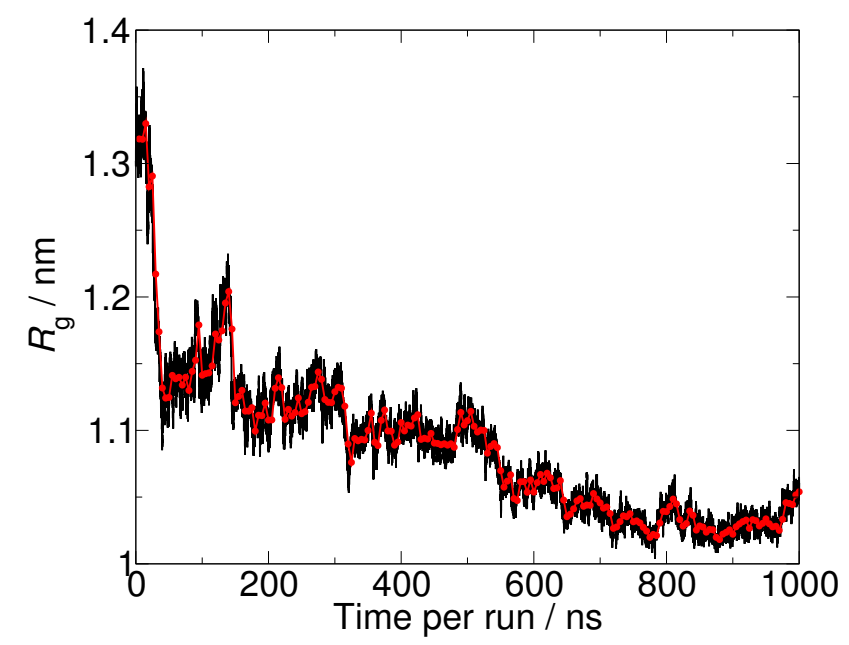

Fig. 3: Average radius of gyration as a function of the simulation time. Data obtained from averaging 15 independent runs of 1000 ns each. All data points (black line) and block-averaged data using a block size of 5 ns (red line).

A formal determination of the equilibration time can be carried out by applying a statistical test. At equilibrium, the $R_{\mathrm{g}}$ signal should show no trends. In the present work, the MK test $[36,37]$ was carried out to check this. The MK test does not require that the data have any particular distribution, but it only holds for uncorrelated data. It was, therefore, applied here to the block-averaged signal of the 15 combined runs, see Fig. 3.

In the MK test, the null hypothesis is that there is no trend in the data. The null hypothesis is rejected or accepted by analyzing the statistic $S$ and its variance $\operatorname{VAR}(S)$. To compute $S$, we conducted a pairwise comparison of all data points with all previous values. Then, $S$ is given by adding +1 for a positive difference, -1 for a negative difference, and 0 for tie values. If the final $S$ is exactly zero, no trend can be assumed with a confidence of $100 \%$, i.e., the null hypothesis is accepted. A large positive or large negative $S$ indicates an increase or a decrease in the data, and therefore the null hypothesis is rejected. However, the magnitude of $S$ depends on the number of points in the studied dataset. Instead of evaluating directly $S$, a normalized value of $S$ is used that account for the variance of the data. From the analysis of the normalized value of $S$, the so-called p-value is obtained, which gives the probability of erroneously rejecting the null hypothesis. More details are given in Ref. [36, 37].

The MK test was carried out iteratively and in reverse order, i.e., subsets of blockaveraged $R_{\mathrm{g}}$ were generated starting from the last point in the data set $(t=1000$ ns) and proceeding to earlier times. Then, the MK test is carried out for each subset. Statistical analysis in reverse order is an effective method to locate the equilibration time in MD simulations [33], and the main idea is the following. As the number of points in the subset increases, the border between the non-equilibrated and the 
equilibrated region is crossed. When this border is crossed, a trend can be detected for all following subset.

The MK test shows that there is a systematic trend in the data for $t<670$ ns. The probability of erroneously rejecting the null hypothesis is below 0.05 in that time span, and even below $10^{-7}$ for $t<600 \mathrm{~ns}$.

\subsection{Convergence of single runs}

Based on the analysis described above, only data for $t>670 \mathrm{~ns}$ was used to calculate the average $R_{\mathrm{g}}$. The results for the 15 runs are presented in Fig. 4 together with their statistical uncertainty obtained from block averaging with a block size of $20 \mathrm{~ns}$. Also, the result obtained from the averaged signal of all runs (see Fig. 3) is presented. Fig. 4 shows that the statistical uncertainty of the results obtained in different runs varies strongly. This is a consequence of the strongly different patterns of the signals shown in Fig. 1.

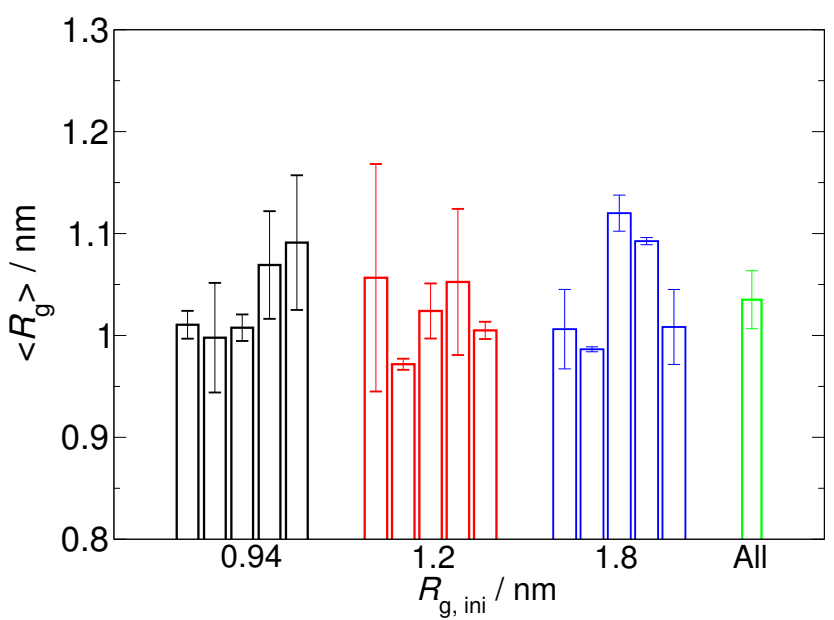

Fig. 4: Average $R_{\mathrm{g}}$ for $t>670 \mathrm{~ns}$ : Results obtained from 15 individual runs started from different initial radius of gyration $R_{\mathrm{g}, \text { ini }}$ and from the arithmetic average of all runs. The error bars were obtained from block averaging.

Fig. 5 shows the probability distribution of $R_{\mathrm{g}}$ for individual runs (panels a-c for the three starting configurations) and the combined runs (panel d), obtained for $t>$ $670 \mathrm{~ns}$. The distributions of $R_{\mathrm{g}}$ for single runs differ strongly. Two main peaks are observed, the first at $R_{\mathrm{g}}=0.98 \mathrm{~nm}$ and the second at $R_{\mathrm{g}}=1.10 \mathrm{~nm}$. The simulations started from the coil and intermediate states trend to populate the first peak at 0.98 $\mathrm{nm}$ while those which were started from the globule state populate the second peak at $1.10 \mathrm{~nm}$. This shows that even for $t>670 \mathrm{~ns}$, the results from individual runs still depend strongly on the initial configuration. Therefore, individual runs are not representative of the equilibrium. Fig. 5 shows the probability distribution obtained by combining the five replicas for each of the three initial states. Also, these distributions differ substantially. Finally, the distribution obtained by combining the fifteen runs is shown in panel (d) of Fig. 5. While this is the best guess for the equilibrium distribution that can be obtained from the present data, the differences with respect to the other panels indicate that it is strongly biased by the choice of the initial conditions. 


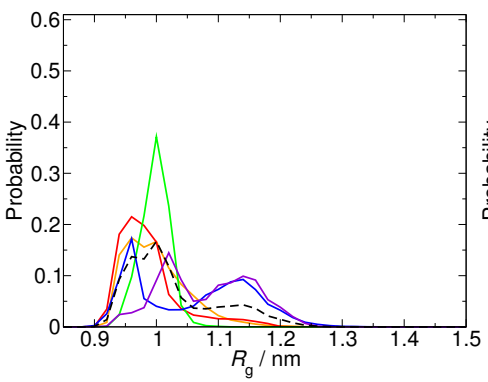

(a)

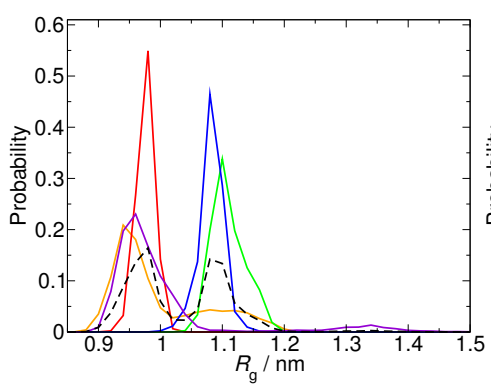

(c)

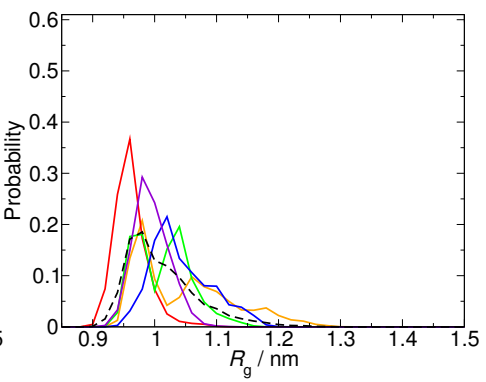

(b)

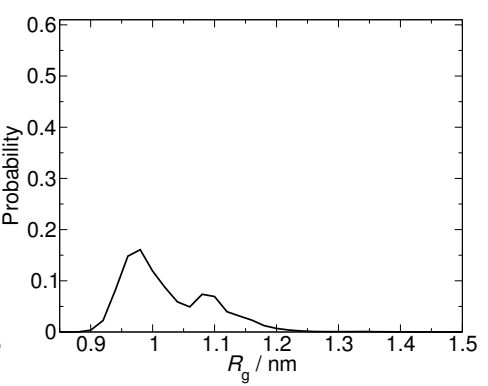

(d)

Fig. 5: Probability distribution of the radius of gyration for $t>670$ ns. Simulations started at (a) $R_{\mathrm{g}, \text { ini }}=0.94 \mathrm{~nm}$, (b) $R_{\mathrm{g}, \text { ini }}=1.20 \mathrm{~nm}$, (c) $R_{\mathrm{g}, \text { ini }}=1.80 \mathrm{~nm}$. Single runs (solid lines) and combined 5 replicas (dashed line). Panel (d) shows the probability distribution of all combined runs.

Obviously, only a part of the conformational space is sampled. Single runs lead to globule conformations that are difficult to convert into other conformations. Globule conformations are tightly packed, and important energetic barriers resulting from steric effects hinder conformational changes. Therefore, a transformation from a given globule state to a new globule state may require the unfolding and refolding of the oligomer. Since the coil state is not favorable for the studied conditions, such transitions are rarely observed. Therefore, single runs sample only part of the globule state.

The GR (Gelman-Rubin) test [32] can be used to check quantitatively if individual runs are representative of the common distribution. In the GR test, the within-replica variance is compared to the variance of the pooled data. If the individual replicas are representative, there should be no significant difference between these variances. Difference in the variances indicates that single replicas have visited only part of the accessible distribution. The GR test was carried out for $R_{\mathrm{g}}$ values obtained for $t>$ 670 ns. Results are presented in Fig. 6 , where the so-called scale reduction factor $R$ is shown as a function of the simulation time. $R$ is the square root of ratio of the pooled variance $V$ and the within-replica $W$ variance. $R$ should drop to 1 if the simulation time is sufficiently large. This is clearly not the case: even for the long simulation time used here, there are important differences between $V$ and $W$ that hardly diminish. 


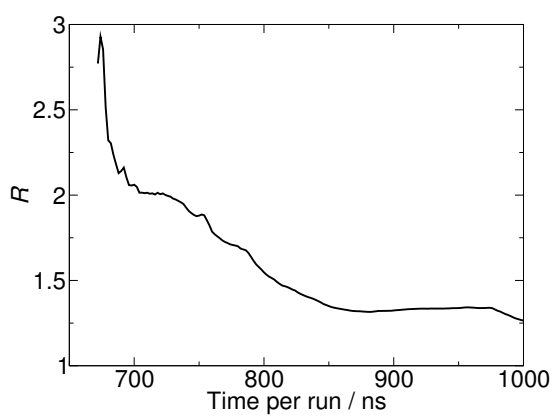

Fig. 6: Gelman-Rubin scale reduction factor $R$ (see text) as a function of the simulation time starting at $t=670 \mathrm{~ns}$. The fact that $R$ does not approach to 1 shows that individual runs are not representative of the pooled distribution.

\section{Conclusions}

The present study shows that direct atomistic MD simulations are not suited for studying equilibrium properties of NIPAM in water, even for the simple case of a single moderate-sized polymer chain. The simulation time that would be needed to sample the configuration space adequately exceeds the presently available computational resources by far. In the present work, fifteen independent simulations of 1000 ns each starting from different initial configurations were conducted. It took about $650 \mathrm{~ns}$ until the radius of gyration of the chains showed no systematic trends, but the data obtained after this long equilibration time is biased by a clear influence of the initial state. The total simulation time of the present study is $15,000 \mathrm{~ns}$. It is so far below the time that would be required for obtaining reliable equilibrium data and no estimate of that time can be given. Many authors, including our own group, have studied the coil-globule transition of NIPAM in water by all-atom direct MD simulations. Without any exception, these simulations are not suited for making safe statements on the thermodynamic equilibrium properties of the studied system.

Even though only a specific situation was studied here, it may be expected that the basic findings also hold for other studies that aim at determining equilibrium properties of polymers in solution by brute force atomistic MD simulation. The results underpin the challenges in such simulations and highlight the necessity of enhanced sampling techniques [54] and workarounds, such as simplified scenarios [25] or coarsegraining [55], for such studies.

\section{Acknowledgment}

The authors acknowledge financial support from the Reinhart Koselleck Program (HA1993/15-1) of the German Research Foundation (DFG) and the German Federal Ministry of Education and Research (BMBF) within the project TalPas (01IH16008F). The present work was conducted under the auspices of the Boltzmann-Zuse Society of Computational Molecular Engineering (BZS). The simulations were carried out on Elwetritsch at the Regional University Computing Center Kaiserslautern (RHRK) under the grant TUK-TLMV, and on SuperMUC at the Leibniz Supercomputing Center (LRZ), Garching, under the grant SPARLAMPE (pr48te). 


\section{Author contribution statement}

E.J.G did the MD simulations. E.J.G. and H.H. contributed equally to the analysis of the data and preparation of the manuscript.

\section{References}

1. A. Grosberg, A. Khokhlov, Statistical Physics of Macromolecules (AIP Press, New York 1994)

2. S.M. Mel'nikov, V.G. Sergeyev, K. Yoshikawa, J. Am. Chem. Soc. 117(9), 2401 (1995)

3. D. Ito, K. Kubota, Macromolecules 30(25), (1997) 7828

4. M. Alaghemandi, S. Eckhard, Macromol. Theory Simul. 21(2), 106 (2011)

5. S.A. Deshmukh, S.K.R.S. Sankaranarayanan, K. Suthar, D.C. Mancini, J. Phys. Chem. B 116(9), 2651 (2012)

6. S.A. Deshmukh, Z. Li, G. Kamath, K.J. Suthar, S.K.R.S. Sankaranarayanan, D.C. Mancini, Polymer 54(1), 210 (2013)

7. V. Boţan, V. Ustach, R. Faller, K. Leonhard, J. Phys. Chem. B 120(13), 3434 (2016)

8. G. Longhi, F. Lebon, S. Abbate, S.L. Fornili, Chem. Phys. Lett. 386(1), 123 (2004)

9. F. Gangemi, G. Longhi, S. Abbate, F. Lebon, R. Cordone, G.P. Ghilardi, S.L. Fornili, J. Phys. Chem. B 112(38), 11896 (2008)

10. J. Walter, V. Ermatchkov, J. Vrabec, H. Hasse, Fluid Phase Equilibria 296(2), 164 (2010)

11. H. Du, R. Wickramasinghe, X. Qian, J. Phys. Chem. B 114(49), 16594 (2010)

12. E. Chiessi, A. Lonardi, G. Paradossi, J. Phys. Chem. B 114(25), 8301 (2010).

13. Y. Kang, H. Joo, J.S. Kim, J. Phys. Chem. B 120(51), 13184 (2016)

14. T.E. de Oliveira, D. Mukherji, K. Kremer, P.A. Netz, J. Chem. Phys. 146(3), 034904 (2017)

15. T.E. de Oliveira, C. M. Marques, P. A. Netz, Phys. Chem. Chem. Phys. 20(15), 10100 (2018)

16. F. Afroze, E. Nies, H. Berghmans, J. Mol. Struct. 554(1), 55 (2000)

17. R. Gomes de Azevedo, L.P.N. Rebelo, A.M. Ramos, J. Szydlowski, H.C. de Sousa,

J. Klein, Fluid Phase Equilibria 185(1), 189 (2001)

18. A. Milewska, J. Szydlowski, L.P.N. Rebelo, J. Polymer Sci. Part B: Polym. Phys. 41(11), 1219 (2003)

19. R. Singh, S.A. Deshmukh, G. Kamath, S.K.R.S. Sankaranarayanan, G. Balasubramanian, Comp. Mater. Sci. 126, 191 (2017)

20. J.B. Clarage, T. Romo, B.K. Andrews, B.M. Pettitt, G.N. Phillips, Proc. Natl Acad. Sci. 92(8), 3288 (1995)

21. S. Genheden, U. Ryde, Phys. Chem. Chem. Phys. 14(24), 8662 (2012)

22. L. Sawle, K. Ghosh, J. Chem. Theory Comput. 12(2), 861 (2016)

23. K. Lindorff-Larsen, S. Piana, R.O. Dror, D.E. Shaw, Science 334(6055), 517 (2011)

24. C. Andrea, F. Philippe, C. Amedeo, Proteins Struct. Funct. Bioinforma. 47(3), 305 (2002)

25. E.J. García, D. Bhandary, M.T. Horsch, H. Hasse, J. Mol. Liq. 268, 294 (2018)

26. C. Dalgicdir, F. Rodriguez-Ropero, N.F. van der Vegt, J. Phys. Chem. B 121, 7741 (2017)

27. R.C. Bernardi, M.C. Melo, K. Schulten, Biochim. Biophys. Acta BBA-Gen. Subj. $\mathbf{1 8 5 0}(5), 872(2015)$

28. M. Saunders, K.N. Houk, Y.D. Wu, W.C. Still, M. Lipton, G. Chang, W.C. Guida, J. Am. Chem. Soc. 112(4), 1419 (1990)

29. T. Kawai, N. Tomioka, T. Ichinose, M. Takeda, A. Itai, Chem. Pharm. Bull. 42(6), 1315 (1994)

30. I.T. Christensen, F.S. Jørgensen, J. Comput. Aided Mol. Des. 11(4), 385 (1997)

31. S.K. Schiferl, D.C. Wallace, J. Chem. Phys. 83(10), 5203 (1985)

32. S.P. Brooks, A. Gelman, J. Comput. Graph. Stat. 7(4), 434 (1998) 
33. W. Yang, R. Bitetti-Putzer, M. Karplus, J. Chem. Phys. 120(6), 2618 (2004)

34. A. Grossfield, D.M. Zuckerman, Annu. Rep. Comput. Chem. 5, 23 (2009)

35. T.D. Romo, A. Grossfield, J. Chem. Theory Comput. 7(8), 2464 (2011)

36. H.B. Mann, Econometrica 13(3), 245 (1945)

37. M.G. Kendall, Rank correlation methods (C. Griffin, London, UK, 1975)

38. J. Kstner, W. Thiel, J. Chem. Phys. 124(23), 234106 (2006)

39. L.S. Caves, J.D. Evanseck, M. Karplus, Protein Sci. 7(3), 649 (1998)

40. E. Chiessi, G. Paradossi, J. Phys. Chem. B 120(15), 3765 (2016)

41. S.W.I. Siu, K. Pluhackova, R.A. Bckmann, J. Chem. Theory Comput. 8(4), 1459 (2012)

42. G. Bussi, D. Donadio, M. Parrinello, J. Chem. Phys. 126(1), 014101 (2007)

43. H.J.C. Berendsen, J.P.M. Postma, v.W.F. Gunsteren, A. DiNola, J.R. Haak, J. Chem.

Phys. 81(8), 3684 (1984)

44. M. Parrinello, A. Rahman, J. Appl. Phys. 52(12), 7182 (1981)

45. T. Darden, D. York, L. Pedersen, J. Chem. Phys. 98(12), 10089 (1993)

46. W.L. Jorgensen, D.S. Maxwell, J. Tirado-Rives, J. Am. Chem. Soc. 118(45), 11225 (1996)

47. H.J.C. Berendsen, J.R. Grigera, T.P. Straatsma, J. Phys. Chem. 91(24), 6269 (1987).

48. B. Hess, H. Bekker, H.J.C. Berendsen, J.G.E.M. Fraaije, J. Comput. Chem. 18(12), 1463 (1997)

49. F.K. Anton, H. Berk, H.J.C. Berendsen, J. Comput. Chem. 20(8), 786 (1999)

50. B. Loubet, W. Kopec, H. Khandelia, J. Chem. Theory Comput. 10(12), 5690 (2014)

51. K. Olesen, N. Awasthi, D.S. Bruhn, W. Pezeshkian, H. Khandelia, J. Chem. Theory Comput. 14(6), $3342(2018)$

52. D. Van Der Spoel, E. Lindahl, B. Hess, G. Groenhof, A.E. Mark, H.J.C. Berendsen, J. Comput. Chem. 26(16), 1701 (2005)

53. M.J. Abraham, T. Murtola, R. Schulz, S. Páll, J.C. Smith, B. Hess, E. Lindahl, SoftwareX 1-2, 19 (2015)

54. V. Palivec, D. Zadrazil, J. Heyda, ArXiv e-prints 1806, arXiv:1806.05592 (2018)

55. D. Mukherji, K. Kremer, Macromolecules 46(22), 9158 (2013) 